\title{
EFICIÊNCIA DE ÍNDICES DE RISCO DE INCÊNDIOS PARA O PANTANAL SUL-MATO-GROSSENSE
}

\author{
EFFICIENCY OF FIRE RISK INDICES FOR PANTANAL SUL-MATO-GROSSENSE
}

\author{
Balbina Maria Araújo Soriano ${ }^{1}$ Omar Daniel ${ }^{2}$ Sandra Aparecida Santos ${ }^{3}$
}

\begin{abstract}
RESUMO
O objetivo deste trabalho foi comparar os índices de risco de incêndios a partir de focos de calor e definir o mais eficiente para as condições do Pantanal Sul-mato-grossense, com base em dados meteorológicos e número de focos de calor. O trabalho consistiu na análise de correlação entre variáveis meteorológicas e a ocorrência dos focos de calor, bem como a comparação entre os índices de risco de incêndios: Angströn (B), Telicyn (I), Nesterov (G), Monte Alegre (FMA) e Monte Alegre Modificada (FMA ${ }^{+}$). Foram utilizados dados meteorológicos coletados às 14 horas na estação Climatológica Principal de Nhumirim e de focos de calor da passagem noturna do NOAA (12 e 15), no período de 1999 a 2008 . A velocidade do vento e a umidade relativa do ar foram as variáveis meteorológicas que apresentaram maior correlação com a ocorrência de focos de calor. Para detecção de qualquer grau de risco de incêndio, a FMA, o G e a FMA foram os índices mais eficientes. Para a detecção de graus de risco de incêndio nas classes Muito alto e Alto, o $\mathrm{G}$ foi o mais eficiente, seguido da FMA. Considerando a alta probabilidade de acerto na detecção de riscos de incêndio em qualquer grau e também nas classes de Alto risco a mais, a FMA pode ser considerada a mais adequada para estimar o risco de ocorrência de incêndios para a sub-região da Nhecolândia.
\end{abstract}

Palavras-chave: fórmula de Monte Alegre; queimada; focos de calor; variáveis meteorológicas.

\begin{abstract}
The objective of this work was to compare the indices of fire risk from hotspots and define the most efficient for the conditions of South Pantanal of Mato Grosso, based on meteorological data and number of hotspots. The work consisted of correlation analysis between some meteorological variables and the occurrence of hotspots as well as the comparison between the fire risk indices of Angstron (B), Logarithmic index of Telicyn (I), Nesterov Index (G), Monte Alegre Index (FMA) and Modified Monte Alegre Index (FMA ${ }^{+}$. Meteorological data were collected at 14 hours in the Nhumirim climatological station and hotspots during night of the NOAA12 and NOAA15 from 1999 to 2008. The wind speed and relative humidity were the meteorological variables which best correlated with the occurrence of fire. To detect any degree of fire risk, the FMA, the $\mathrm{G}$ and $\mathrm{FMA}^{+}$were the most efficient indices. For the detection of degrees of fire risk classes very high and high, the $\mathrm{G}$ was the most efficient, followed by the FMA. Considering the high probability of success in detecting fire risks in any degree and also in the class of the most high risk, the FMA can be deemed more appropriate for estimating the fire risk for Nhecolândia sub-region.
\end{abstract}

Keywords: Monte Alegre index; forest fires; hotspots; meteorological variables.

1 Meteorologista, Dr ${ }^{\mathrm{a}}$., Pesquisadora da Embrapa Pantanal, Caixa Postal 109, CEP 79320-900, Corumbá ( MS), Brasil. balbina.soriano@embrapa.br

2 Engenheiro Florestal, Dr., Faculdade de Ciências Agrárias, Universidade Federal da Grande Dourados, Caixa Postal 533, CEP 79804-970 Dourados (MS). omardaniel@ufgd.edu.br

3 Zootecnista, Dr ${ }^{\mathrm{a}}$., Pesquisadora da Embrapa Pantanal, Caixa Postal 109, CEP 79320-900, Corumbá (MS), Brasil. sandra.santos@embrapa.br

Recebido para publicação em 8/08/2010 e aceito em 29/04/2014

Ci. Fl., v. 25, n. 4, out.-dez., 2015 


\section{INTRODUÇÃO}

A criação de bovinos de corte é a principal atividade econômica do Pantanal há mais de dois séculos, pois a região possui extensas áreas de pastagens naturais que são favoráveis a esta atividade. O manejo das pastagens naturais é complexo e dinâmico, em razão da grande variedade de unidades de paisagem (fitofisionomias), que variam espacial e temporalmente, principalmente em função das condições climáticas (SANTOS et al., 2008). Muitas dessas fitofisionomias são propensas a incêndios que podem ocorrer acidentalmente ou serem provocados por práticas de manejo inadequadas de queimas em pastagem.

A ocorrência e a propagação dos incêndios florestais estão fortemente associadas às condições climáticas. A intensidade de um incêndio e a velocidade com que ele se propaga estão diretamente ligadas à umidade relativa, à temperatura e à velocidade do vento. A utilização de dados meteorológicos de boa qualidade é, portanto, vital para o planejamento de prevenção e combate aos incêndios florestais (NUNES et al., 2006).

Desde 2000, a Embrapa Pantanal tem um programa de monitoramento das variáveis meteorológicas e das ocorrências de incêndios no Pantanal Mato-Grossense (SORIANO et al, 2008), onde pode ser observado que, dependendo da variação do clima entre os anos, ocorre maior ou menor número de eventos, modificando a paisagem local.

Em 2005, ano considerado "seco" no Pantanal, em decorrência das chuvas que ficaram abaixo da média histórica, o número de incêndios foi 6.199, o maior número de ocorrência desde 2000 (PADOVANI, 2006). Além da influência do clima no aumento dos incêndios, outro fator importante foi a grande quantidade de material combustível em algumas fitofisionomias que, associado à longa estiagem e baixos índices de umidade relativa do ar, favoreceu grandes incêndios, o que afetou drasticamente o ecossistema da região.

Dentre as medidas preventivas de combate aos incêndios florestais, a utilização de um índice de perigo confiável é de fundamental importância dentro de um plano de prevenção e combate, por permitir a avaliação dos riscos, possibilitando a adoção de medidas preventivas em bases mais eficientes e econômicas (SOARES, 1985; BATISTA, 2004; NUNES et al., 2007).

A estrutura dos índices de perigo de incêndio disponíveis é baseada fundamentalmente na relação entre os incêndios florestais e os elementos meteorológicos (temperatura e umidade do ar, velocidade do vento e precipitação). Seus resultados refletem, antecipadamente, a probabilidade de ocorrer um incêndio, assim como a facilidade de propagação, de acordo com as condições atmosféricas do dia ou da sequência de dias (SOARES, 1972; SOARES e BATISTA, 2007).

A importância destes índices está ligada à prevenção de incêndios, pois é mais vantajoso evitá-los ou mesmo eliminá-los imediatamente após o início do que combatê-lo depois de estabelecido e propagado (MARTINI et al., 2007). Assim podem ser minimizados os impactos causados pelas queimadas, que provocam aumento das concentrações de gases de efeito estufa e aerossóis, causando mudanças na atmosfera e provavelmente no clima do planeta, como também problemas na economia e na saúde da população local.

O objetivo deste trabalho foi comparar os índices de risco de incêndios e definir o mais eficiente para as condições do Pantanal Sul-mato-grossense, tendo como base dados diários meteorológicos da estação climatológica principal de Nhumirim e o número de focos de calor ocorridos na sub-região da Nhecolândia, no período de 1999 a 2008.

\section{MATERIAL E MÉTODOS}

Este estudo foi realizado utilizando-se uma série temporal de 10 anos (1999-2008) de dados de precipitação pluvial diária $(\mathrm{mm})$; temperatura do ar $\left({ }^{\circ} \mathrm{C}\right)$, umidade relativa do ar $(\%)$ e a velocidade do vento $\left(\mathrm{m} \mathrm{s}^{-1}\right)$, medidos às 14 horas (horário padrão das estações climatológicas principais brasileiras), coletados na estação Climatológica Principal de Nhumirim (latitude 1859'21'S, longitude $56^{\circ} 37^{\prime} 25^{\prime}$ 'W, altitude $102 \mathrm{~m}$ ), localizada na fazenda Nhumirim, situada na sub-região da Nhecolândia no Pantanal Sul-mato-grossense.

Asub-região da Nhecolândia (latitude $18^{\circ} 10^{\prime}$ e $19^{\circ} 25^{\prime} \mathrm{S}$, longitude $55^{\circ} 5^{\prime}$ e $\left.57^{\circ} 18^{\prime} \mathrm{W}\right)$ enquadrase no tipo climático Aw (Köppen), definido como tropical, megatérmico, com a temperatura média do mês mais frio superior a $18^{\circ} \mathrm{C}$. As médias anuais de precipitação, temperatura e umidade relativa do ar, no período de 1977-2001, foram $1.181 \mathrm{~mm}$, $25,4^{\circ} \mathrm{C}$ e $81,3 \%$, respectivamente (SORIANO e ALVES, 2005). O regime hídrico apresenta dois períodos distintos: um chuvoso, que se inicia em outubro e estende-se até março, quando ocorrem 
aproximadamente $80 \%$ do total anual de chuvas e outro com poucas chuvas, de abril a setembro (CAMPELO JUNIOR et al., 1997).

Os solos são arenosos em sua maioria, com textura fina, mas eventualmente, são encontradas manchas de solos siltosos ou argilosos. O relevo é plano, com altitude aproximada de $90 \mathrm{~m}$ acima do nível do mar (RATTER et al., 1988) apresentando pequenos desníveis altimétricos (até $3 \mathrm{~m}$ ) entre as fitofisionomias.

A área deste estudo foi definida em função da representatividade dos dados coletados nesta estação que, segundo a World Meteorological Organization - WMO (2008), deve abranger um raio de $100 \mathrm{~km}$ e apresentar horizontes amplos, ou seja, não podem ter barreiras que impeçam incidência da radiação solar ou que modifiquem o vento. Analisando-se as cotas topográficas da região, observa-se que a uma distância de $76,6 \mathrm{~km}$, rumo $69^{\circ} \mathrm{NO}$, existe um conjunto de elevações com cota máxima de $150 \mathrm{~m}$ e na direção $\mathrm{W}$ atinge a morraria de Urucum com cotas de aproximadamente 1.040 $\mathrm{m}$ nas proximidades. Assim, para definir a área representativa para os dados desta estação, foi traçada uma linha que cortou o círculo de raio $100 \mathrm{~km}$ de norte a sul, passando pelo ponto de cruzamento do rumo citado em um raio de $75 \mathrm{~km}$ (UTM X = 469.969,7646), procurando o distanciamento da influência dos morros da região (Figura 1).

Foram também utilizados dados diários de focos de calor no período de 1999 a 2008, disponibilizados pela Divisão de Processamento de Imagens/Instituto Nacional de Pesquisas Espaciais para toda a América Latina, no formato de tabela com coordenadas, que pode ser convertido em mapa de pontos, georreferenciado, ou no formato de mapa shapefile (INPE, 2008). Os dados analisados foram aqueles gerados pelo satélite NOAA12-noturno e o NOAA15-noturno, devido à desativação do NOAA12-noturno a partir de 10 de julho de 2007.

Os focos de calor são identificados a partir de elementos de resolução espacial (pixels) que apresentam altas temperaturas, os quais possuem os mais baixos valores de nível de cinza nas imagens da região infravermelho termal banda $3(3.7 \mu \mathrm{m})$ do AVHRR. Esta banda mede emissão de energia radiante da superfície terrestre, na qual os pixels saturados correspondem à temperatura de pelo menos $47^{\circ} \mathrm{C}$, normalmente associada a alvos em combustão (INPE, 2009).

Os dados das ocorrências diárias de focos de calor e suas respectivas coordenadas foram trabalhados em planilha eletrônica, com o objetivo de eliminar os focos que estivessem fora da área de abrangência (Figura 1), isto é, raio maior que 100 $\mathrm{km}$ e além da linha vertical próxima aos morros. Para executar esta rotina, foi usado o principio do teorema de Pitágoras, calculando-se a distância do foco de calor à estação de Nhumirim, eliminandose posteriormente os dados com raios além de 100 $\mathrm{km}$. Simultaneamente, foram eliminados os pontos que ficaram além das coordenadas UTM sentido oeste, cujos valores $\mathrm{X}$ tenham sido menores do que $469.969,7646 \mathrm{~m}$. Uma nova tabela foi gerada somente com o número de focos de incêndio diários que ocorreram no interior da área de abrangência mostrada na Figura 1.

Para verificar a relação entre o total mensal

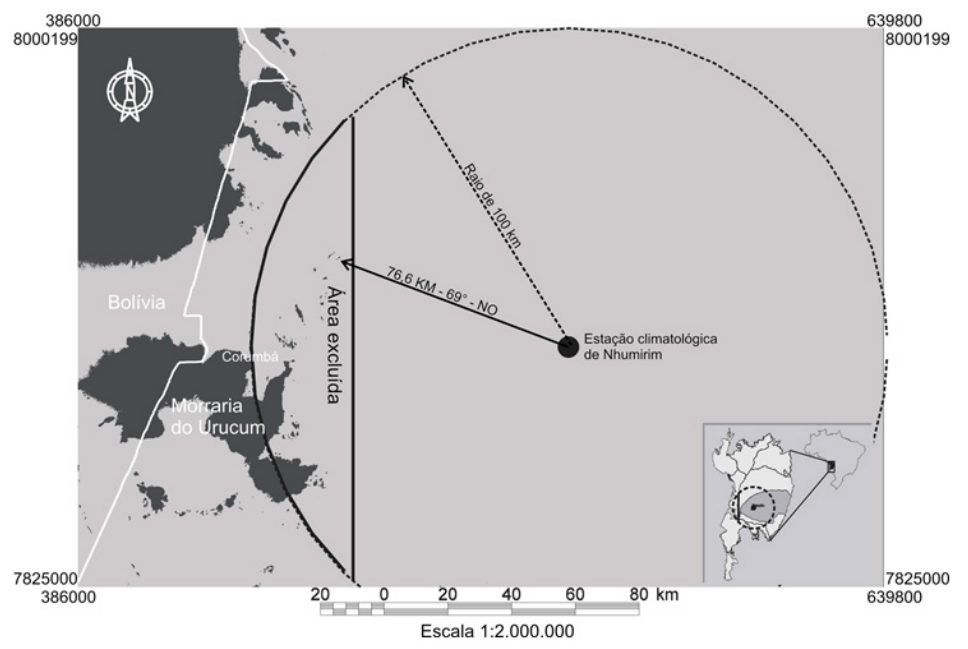

FIGURA 1: Área do estudo definida segundo as recomendações da WMO (2008). FIGURE 1: Study area defined according to the WMO (2008) recommendations. 
de focos de incêndio (variável dependente) e valores médios mensais das variáveis meteorológicas, foram realizadas análises de correlação e regressão exponencial.

Para comparação entre índices de risco de incêndios foram utilizados os Índices de Angströn (B), Índice Logarítmico de Telicyn (I), Índice de Nesterov (G), Fórmula de Monte Alegre (FMA) e Fórmula de Monte Alegre Modificada $\left(\mathrm{FMA}^{+}\right)$, calculados diariamente durante o período de 1999 a 2008, por meio de metodologia proposta por Nunes (2005) e Soares (1972).

Como os índices são divididos em não acumulativos (baseiam-se inteiramente nas condições correntes do dia) e acumulativos (consideram efeitos passados do tempo), cujos resultados são interpretados por meio de diferentes escalas, surgiu a dificuldade de comparação direta entre eles em igualdade de condições. Decidiu-se então fazer a comparação em duas etapas:

$1^{\text {a }}$ - Foi verificada a probabilidade da detecção dos focos de calor registrados pelos cinco índices, em condições favoráveis e desfavoráveis. Para isto, utilizou-se dos valores diários estimados de cada índice e suas respectivas escalas nos dias que ocorreram os focos, isto é, se eles indicavam ou não a existência de perigo de incêndio.

$2^{\mathrm{a}}$ - Foi feita a comparação entre os quatro índices acumulativos, utilizando-se do artifício proposto por Soares e Paz (1973) e Soares (1987), no qual os valores apresentados pelos índices nos dias de ocorrência de focos de calor foram convertidos em porcentagens dos valores máximos obtidos durante o período de observação. Assim, obteve-se a eficiência de cada índice em uma escala única, tendo sido então possível comparálos entre si. Os índices foram ordenados de acordo com suas eficiências em cada ocorrência de focos de calor e submetidos ao teste de Bartlett para verificar a homogeneidade das variâncias ao nível de significância $p<0,01$. As comparações foram feitas pelo teste não paramétrico de Friedman, utilizando software ASSISTAT (SILVA, 1996). Este teste ordena os resultados para cada um dos casos e em seguida calcula a média das ordens para cada amostra. Se não existirem diferenças entre as amostras, as médias das ordens devem ser similares. A estatística deste teste tem uma distribuição $\chi^{2}$.

\section{RESULTADOS E DISCUSSÃO}

Os resultados indicaram que a $\mathrm{VV}$ $(\mathrm{r}=0,79)$ e UR $(\mathrm{r}=-0,69)$ são as variáveis que melhor se correlacionam com o número de ocorrência de focos de calor. A regressão com base em modelo exponencial resultou que as variáveis independentes VV e UR apresentaram melhor ajuste do que TEMP e PP (Tabela 1).

Os resultados das correlações entre as variáveis meteorológicas e o número de focos de calor foram os esperados para a sub-região da Nhecolândia, já que a maior concentração desses focos se dá entre os meses de agosto e novembro, principalmente em agosto e setembro, quando ocorrem os mais baixos índices de umidade relativa, concomitantemente com os maiores valores de velocidade do vento.

A influência da precipitação pluvial e da temperatura do ar sobre as ocorrências dos focos de calor não se mostrou eficaz isoladamente através da correlação linear, sugerindo estudos mais detalhados por meio da combinação de uma ou mais variáveis.

A baixa correlação entre a precipitação pluvial e o número de focos de calor não implica

TABELA 1: Correlação linear de Pearson (r) e coeficiente de determinação para regressão exponencial $\left(\mathrm{R}^{2}\right)$ entre o total mensal do número de focos de calor (variável dependente) e a média mensal da precipitação pluvial (PP), umidade relativa do ar (UR), temperatura do ar (TEMP) e a velocidade do vento (VV).

TABLE 1: Linear correlation of Pearson ( $\mathrm{r}$ ) and coefficient of determination for exponential regression $\left(\mathrm{R}^{2}\right)$ among the total monthly from the number hot spot and mean monthly precipitation (PP), relative humidity (RH), air temperature (TEMP) and wind speed (VV).

\begin{tabular}{ccc}
\hline Variáveis meteorológicas & $\mathrm{r}$ & $\mathrm{R}^{2}$ \\
\hline $\mathrm{VV}\left(\mathrm{m} \mathrm{s}^{-1}\right)$ & 0,7941 & 0,8491 \\
$\mathrm{UR}(\%)$ & $-0,6934$ & 0,6141 \\
$\mathrm{TEMP}\left({ }^{\circ} \mathrm{C}\right)$ & 0,4418 & 0,2361 \\
$\mathrm{PP}(\mathrm{mm})$ & $-0,2577$ & 0,1124 \\
\hline
\end{tabular}


que a chuva não tenha influência na ocorrência de incêndios, pois, segundo Soares e Paz (1973) e Soares (1985), a precipitação é de suma importância para a caracterização do clima, estando a ocorrência dos incêndios sempre associada a períodos sem chuva. Existe uma forte correlação entre ocorrência de grandes incêndios e prolongados períodos de seca.

Nesse contexto, a precipitação é, sem dúvida, a variável meteorológica capaz de alterar com maior rapidez as condições de umidade dos combustíveis florestais mortos, sendo fundamental a atenção com esta variável.

Dentro da área de abrangência dos dados climáticos foram totalizados 7.157 focos de calor no período analisado. Os valores dos índices calculados nos dias que ocorreram esses focos constituíram os dados básicos para efetuar as comparações entre eles.

$\mathrm{Na} 1^{\text {a }}$ etapa da metodologia proposta, calculou-se a probabilidade de acerto dos focos de calor registrados pelos cinco índices testados (Tabela 2). A ocorrência de focos de calor em condições desfavoráveis significa a existência de foco em dias nos quais os índices não acusaram riscos, ou seja, o grau de perigo foi nulo.

Observa-se que a FMA registrou a ocorrência de perigo de incêndio em 691 dos 697 dias em que se registraram os focos de calor no período estudado. A FMA, portanto, mostrouse mais eficiente para indicar a probabilidade de incêndios, já que apresentou $99 \%$ de acerto nos focos de calor, seguido do índice de G (98\%), da $\mathrm{FMA}^{+}(97 \%)$, do I (87\%) e B (84\%).

O índice de Angströn (B), além de ter obtido a menor probabilidade de detecção a algum grau de perigo de incêndios nos dias quando se registrou foco de calor, também foi o que apresentou a maior probabilidade de detecção em dias que não houve nenhum foco, demonstrando ser o menos eficiente para a sub-região da Nhecolândia.

Devido à FMA, $\mathrm{FMA}^{+}$, I e G apresentarem uma escala com quatro ou mais graus de perigo de incêndio, sendo um deles nulo, separou-se o resultado da detecção de focos registrados sob condições favoráveis em quatro graus de perigo, permitindo assim uma comparação mais detalhada (Tabela 3).

Verifica-se que o I teve boa probabilidade em acertar o maior número de focos de calor no grau Alto ou grande de perigo (69\%), sendo baixíssima sua probabilidade de ocorrência nas classes Pequeno e Médio. $\mathrm{A} \mathrm{FMA}^{+}$teve tendência semelhante ao I, mas concentrou a probabilidade de detectar o maior número de focos no grau Muito alto ou altíssimo.

O G e FMA tiveram a probabilidade de detectar os maiores números de focos nos graus Alto e Muito alto (G: $18 \%$ e $77 \%$ e FMA: $22 \%$ e $64 \%$, respectivamente), sendo que a FMA foi o índice que apresentou a probabilidade de ocorrer o maior número de focos no grau Médio (10\%). Deppe et al. (2004) também registraram que FMA é capaz de registrar grande percentual de acerto nos riscos elevado e extremo.

Se for considerado apenas o somatório dos graus de perigo de incêndios Alto e Muito alto, as probabilidades de detecção foram maiores para o $\mathrm{G}$ $=95 \%, \mathrm{FMA}=86 \%, \mathrm{FMA}^{+}=83 \%$ e I $=69 \%$.

$\mathrm{Na} 2^{\mathrm{a}}$ etapa da metodologia proposta, os valores máximos dos quatro índices acumulativos em toda série de dados considerada foram FMA = $303,62, \mathrm{FMA}^{+}=9,04862 \mathrm{E}+15, \mathrm{I}=133,38$ e $\mathrm{G}=$ $112.450,2$. Dividiu-se o valor calculado nos dias que ocorreram focos pelos respectivos valores máximos

TABELA 2: Probabilidade (P) da ocorrência de focos de calor registrados (In) pelos índices: Fórmula de Monte Alegre (FMA), Fórmula de Monte Alegre Modificada $\left(\mathrm{FMA}^{+}\right)$, Telicyn (I), Nesterov (G) e Angströn (B), em condições favoráveis e desfavoráveis.

TABLE 2: Probability (P) of occurrence registered fires (In) by the indices: Monte Alegre Index (FMA), Modified Monte Alegre Index (FMA ${ }^{+}$), Telicyn (I), Nesterov (G) and Angstron (B), under favorable and unfavorable conditions.

\begin{tabular}{|c|c|c|c|c|c|c|c|c|c|c|}
\hline & \multicolumn{5}{|c|}{ Focos de calor } & \multicolumn{5}{|c|}{ Probabilidade } \\
\hline Condições do dia segundo os índices & (FMA) & $\left(\mathrm{FMA}^{+}\right)$ & (I) & (G) & (B) & (FMA) & $\left(\mathrm{FMA}^{+}\right)$ & (I) & (G) & (B) \\
\hline Há perigo [P(In/condições favoráveis)] & 691 & 678 & 609 & 685 & 587 & 0,99 & 0,97 & 0,87 & 0,98 & 0,84 \\
\hline Há perigo [P(In/condições desfavoráveis $)]$ & 6 & 19 & 88 & 12 & 110 & 0,01 & 0,03 & 0,13 & 0,02 & 0,16 \\
\hline $\mathrm{N}^{0}$ total de focos de calor & 697 & 697 & 697 & 697 & 697 & 1,00 & 1,00 & 1,00 & 1,00 & 1,00 \\
\hline
\end{tabular}


TABELA 3: Probabilidade de ocorrência dos focos de calor registrados pelos índices: Fórmula de Monte Alegre (FMA), Fórmula de Monte Alegre Modificada (FMA ${ }^{+}$), Telicyn (I) e Nesterov (G), em condições favoráveis.

TABLE 3: Probability of occurrence registered fires by the indices: Monte Alegre Index (FMA), Modified Monte Alegre Index (FMA ${ }^{+}$), Telicyn (I) and Nesterov (G), under favorable conditions.

\begin{tabular}{lcccccccc}
\hline \multirow{2}{*}{ Graus de perigo } & \multicolumn{3}{c}{ Focos de calor } & \multicolumn{5}{c}{ Probabilidade } \\
\cline { 2 - 10 } & $(\mathrm{FMA})$ & $\left(\mathrm{FMA}^{+}\right)$ & $(\mathrm{I})$ & $(\mathrm{G})$ & $(\mathrm{FMA})$ & $\left(\mathrm{FMA}^{+}\right)$ & $(\mathrm{I})$ & $(\mathrm{G})$ \\
\hline Pequeno & 21 & 48 & 65 & 5 & 0,03 & 0,07 & 0,09 & 0,01 \\
Médio & 68 & 46 & 60 & 23 & 0,10 & 0,07 & 0,09 & 0,03 \\
Alto ou grande & 156 & 51 & 479 & 122 & 0,22 & 0,07 & 0,69 & 0,18 \\
Muito alto ou altíssimo & 446 & 533 & - & 535 & 0,64 & 0,76 & - & 0,77 \\
\hline \multicolumn{1}{c}{ Total (Há perigo) } & 691 & 678 & 604 & 685 & 0,99 & 0,97 & 0,87 & 0,98 \\
\hline
\end{tabular}

de cada índice, obtendo-se assim a porcentagem de eficiência de cada um deles, sendo possível então compará-los diretamente. Os valores percentuais de eficiência diária foram ordenados do $1^{\circ}$ ao $4^{\circ}$ lugar, estabelecendo-se assim a posição ocupada para cada um.

Por meio da ordenação dos valores percentuais dos quatro índices obteve-se a probabilidade de ocorrência dos focos em $1^{\circ}, 2^{\circ}, 3^{\circ} \mathrm{e}$ $4^{\circ}$ lugar. Analisando-os separadamente, observou-se que FMA foi o mais eficiente na detecção dos focos, pois em $53,94 \%$ dos eventos esteve em $1^{\circ}$ lugar, seguido de G e I que, no entanto, apresentaram suas maiores pontuações para a $3^{\mathrm{a}}$ posição $(42,18 \%$ e $39,46 \%$, respectivamente) e $\mathrm{FMA}^{+} 94,7 \%$ das ocorrências para a $4^{\mathrm{a}}$ posição (Tabela 4 ).

A partir do resultado de ordenação dos dados, verificou-se pelo teste de homogeneidade de variâncias de Bartlett que a hipótese $H_{0}$ foi rejeitada $(\mathrm{p}<0,01)$, indicando que as variâncias dos índices não são homogêneas (Tabela 5), o que determinou a necessidade da aplicação de um teste não paramétrico para as médias.

Assim, o teste de Friedman foi aplicado para comparar a eficiência de cada índice de risco de incêndio, no qual a hipótese nula foi rejeitada $(p<0,0010)$, indicando diferença significativa entre os índices. Com a Diferença Mínima Significativa (DMS), especificou-se onde estava efetivamente essa diferença, classificando em primeiro lugar FMA, seguido de $G$ e $I$, os quais não diferem estatisticamente e por último $\mathrm{FMA}^{+}$(Tabela 6).

Portanto, tanto pela probabilidade da ocorrência de incêndios (Tabela 5), quanto pelo teste de Friedman (Tabela 6), os resultados foram compatíveis.

\section{CONCLUSÕES}

Com base nos estudos e análises realizados neste trabalho, pode-se concluir que a velocidade do vento e a umidade relativa do ar são as variáveis

TABELA 4: Probabilidade da ocorrência de focos registrados pelos índices: Fórmula de Monte Alegre (FMA), Fórmula de Monte Alegre Modificada $\left(\mathrm{FMA}^{+}\right)$, Telicyn (I) e Nesterov (G).

TABLE 4: Probability of occurrence registered fires by the indices: Monte Alegre Index (FMA), Modified Monte Alegre Index (FMA ${ }^{+}$), Telicyn (I) and Nesterov (G).

\begin{tabular}{ccccc}
\hline & \multicolumn{4}{c}{ Probabilidade de ocorrência dos focos - P(In) } \\
\hline Índices & $1^{\circ}$ lugar & $2^{\circ}$ lugar & $3^{\circ}$ lugar & $4^{\circ}$ lugar \\
\hline FMA & 53,94 & 31,71 & 14,35 & 0 \\
FMA $^{+}$ & 1,43 & 0,43 & 3,44 & 94,7 \\
I & 27,40 & 29,12 & 39,46 & 4,02 \\
G & 20,09 & 37,73 & 42,18 & 0 \\
\hline
\end{tabular}


TABELA 5: Resultados do teste de Bartlett.

TABLE 5: Results from Bartlett's test.

\begin{tabular}{ccc}
\hline Índices & Média & Variância \\
\hline FMA & 1,60402 & 0,52688 \\
FMA $^{+}$ & 3,91392 & 0,17361 \\
I & 2,20086 & 0,79006 \\
G & 2,22095 & 0,57468 \\
\hline
\end{tabular}

Em que: Estatística do teste $\left(\chi^{2}\right): 373,58897$; Valor crítico $(a .=1 \%): 11,34488 ; \chi^{2}>\chi^{2}(1 \%) H_{0}$ foi rejeitada $p<0,01$.

TABELA 6: Resultados do teste de Friedman, usando valores de ordenação para verificar a eficiência dos quatro índices.

TABLE 6: Results from Friedman's test, using ranking values to verify the efficiency of the four indices.

\begin{tabular}{|c|c|c|c|c|c|}
\hline Índices de Risco de Incêndio & Média & \multicolumn{3}{|c|}{ Soma dos postos } & \\
\hline FMA & 1,60402 & $1.128,0$ & a & & \\
\hline $\mathrm{FMA}^{+}$ & 3,91392 & $2.737,0$ & & & c \\
\hline I & 2,20086 & $1.545,5$ & & $\mathrm{~b}$ & \\
\hline G & 2,22095 & $1.559,5$ & & b & \\
\hline
\end{tabular}

Em que: Valores seguidos das mesmas letras não diferem estatisticamente pelo teste de Friedman ao nível de 5\%. Estatística do teste $\mathrm{T}^{2}=1.035,9389$; F-Krit $(a .=1 \%): 3,791 ; \mathrm{T}^{2}{ }_{\mathrm{c}}>\mathrm{T}^{2}(1 \%) H_{0}$ foi rejeitada $\mathrm{p}<$ 0,$001 ;$ DMS $=59,644$

meteorológicas que apresentaram maior correlação com a ocorrência do número de focos de calor. Para detecção de qualquer grau de risco de incêndio, os índices mais eficientes em ordem decrescente são: a Fórmula de Monte Alegre (FMA), o Índice de Nesterov (G) e a Fórmula de Monte Alegre Modificada $\left(\mathrm{FMA}^{+}\right)$. Para a detecção de graus de risco de incêndio nas classes Muito alto e Alto, o Índice de Nesterov $(G)$ é o mais eficiente, seguido da Fórmula de Monte Alegre (FMA).

Considerando-se a alta probabilidade de acerto na detecção de riscos de incêndio em qualquer grau e também nas classes de Alto risco a mais, a Fórmula de Monte Alegre (FMA) pode ser considerada a mais adequada para estimar o risco de ocorrência de incêndio para a sub-região da Nhecolândia.

\section{REFERÊNCIAS BIBLIOGRÁFICAS}

BATISTA, A.C. Detecção de Incêndios Florestais por Satélites. Floresta, Curitiba, v. 34, n. 2, p. 237-241, 2004.

CAMPELO JÚNIOR, J.H. et al. Climatologia.
In: PLANO DE CONSERVAÇÃO DA BACIA DO ALTO PARAGUAI - PCBAP. Diagnóstico dos meios físico e biótico: meio biótico. Brasília, DF: Ministério do Meio Ambiente, 1997, v. 2, p. 298-334.

INPE. INSTITUTO NACIONAL DE PESQUISAS ESPACIAIS. Queimadas: monitoramento de focos. Disponível em: $<$ http://www.dpi.inpe.br/ proarco/bdquei madas/bduc.html $>$. Acesso em: 30 nov. 2008.

DEPPE, F. et al. Comparação de índice de risco de incêndio florestal com focos de calor no estado do Paraná. Floresta, Curitiba. v. 34, n. 2, p.119-126, 2004.

INPE. INSTITUTO NACIONAL DE PESQUISAS ESPACIAIS. O Monitoramento de Queimadas em Tempo Quase-Real do INPE. Disponível em http://sigma.cptec.inpe.br/queimadas/perguntas. html. Acesso em: 25 fev. 2009.

MARTINI, L.; DEPPE, F.; LOHMANN, M. Avaliação temporal de focos de calor no estado do Paraná (1999 a 2006). In: SIMPÓSIO BRASILEIRO DE SENSORIAMENTO REMOTO, 8, 2007, Florianópolis. Anais... Florianópolis, SC, INPE, 
2007. p. 4477-4484.

NUNES, J.R.S. FMA ${ }^{+}$- Um novo índice de perigo de incêndios florestais para o Estado do Paraná - Brasil. 2005. 150 f. Tese (Doutorado em Engenharia Florestal) - Setor de Ciências Agrárias, Universidade Federal do Paraná, Curitiba, 2005.

NUNES, J.R.S. et al. FMA ${ }^{+}$Um Novo Índice de Perigo de Incêndios Florestais para o Estado do Paraná - Brasil. Floresta, Curitiba, v. 36, n. 1, p. 75-91, 2006.

NUNES, J.R.S.; SOARES, R.V.; BATISTA, A.C. Ajuste da Fórmula de Monte Alegre Alterada $\mathrm{FMA}^{+}$para o estado do Paraná. Floresta, Curitiba, v.37, n.1, p.1-14, 2007.

PADOVANI, C.R. Dados focos de calor 2000-2006. Corumbá: Embrapa Pantanal, 2006. Disponível em: $\quad<$ http://www.cpap.embrapa.br/fogo/fogo index.htm>. Acesso em: 25 set. 2009.

RATTER, J.A. et al. Observations on woody vegetation types in the Pantanal and around Corumbá. Notes from the Royal Botanic Garden Edinburgh, v.45, p.503-525, 1988.

SANTOS, S.A. et al. Pecuária no Pantanal: em busca da sustentabilidade. In: Albuquerque, A.C.S. e Silva, A.G. Agricultura Tropical: quatro décadas de inovações tecnológicas. V.II, cap.3, p.535-570, 2008.

SILVA, F. de A.S. e. The ASSISTAT Software: statistical assistance. In: INTERNATIONAL CONFERENCE ON COMPUTERS IN AGRICULTURE, 6, Cancun, 1996. Anais... Cancun: American Society of Agricultural Engineers, 1996. p.294-298.

SOARES, R.V. Determinação de um índice de perigo de incêndios para a região centroparanaense, Brasil. 1972, 72 p. Dissertação (Mestrado em Ciências Florestais) - Instituto Interamericano de Ciências Agrícolas da OEA, Turrialba, Costa Rica.

SOARES, R.V.; PAZ, G. Uma nova fórmula para determinar o grau de perigo de incêndios florestais na região centro-paranaense. Floresta, Curitiba, v. 4 n. 3, p. 15-25, 1973.

SOARES, R.V. Incêndios Florestais: controle e uso do fogo. Curitiba: Fundação de Pesquisas Florestais do Paraná, 1985. 213p.

SOARES, R.V. Comparação entre quatro índices na determinação do grau de perigo de incêndios no município de Rio Branco do Sul - Pr. Floresta, Curitiba, v. 17, n. 12, p. 31-35, 1987.

SOARES, R. V.; BATISTA, A. C. Incêndios Florestais: controle, efeitos e uso do fogo. Curitiba, 2007. $250 \mathrm{p}$.

SORIANO, B.M.A.; ALVES, M.J.M. Boletim Agrometeorológico ano 2002 para a sub-região da Nhecolândia, Pantanal, Mato Grosso do Sul, Brasil. Corumbá: Embrapa Pantanal, 2005. 29 p. (Embrapa Pantanal. Documentos, 76).

SORIANO, B.M.A. et al. Monitoramento dos focos de calor e das variáveis meteorológicas para o Pantanal em 2008. Corumbá: Embrapa Pantanal, 2008. 4 p. (Embrapa Pantanal. Comunicado Técnico, 73).

WMO. WORLD METEOROLOGICAL ORGANIZATION. Guide to Meteorological Instruments and Methods of Observation: Weather, Climate, Water. WMO $\mathrm{n}^{\mathrm{o}} 8$, Seventh edition, Geneva, 2008. 